\title{
Correspondence
}

\section{Use of diving reflex in an infant with supraventricular tachycardia*}

Sir,

The diving reflex is a vagally mediated response to sudden immersion of the face in cold water. It slows the heart, while conserving blood pressure and cerebral blood flow. It has been used to treat refractory supraventricular tachycardia in adults and recently in a child (Whitman and Zakeosian, 1976). We report a further case of its use with effect in an infant.

A 12-week-old girl was admitted with a history of poor feeding and loss of weight. She was found to have a heart rate of 200, tachypnoea, and an enlarged liver. Electrocardiogram (ECG) (Fig. 1) showed a supraventricular tachycardia. Unilateral carotid sinus massage and eyeball pressure failed to correct the rhythm and IM digoxin was started. She remained in severe heart failure with continuing tachycardia for 13 hours, and further attempts to convert her rhythm with eyeball pressure failed.

*See also Short report on p. 515. Ed.
The diving reflex was elicited by occluding her nose and mouth by hand and immersing her face in a sink of iced water for about 4 seconds. She reverted to sinus rhythm immediately. ECG following reversion showed bi-atrial hypertrophy, with a normal P-R interval (Fig. 2). Her subsequent course was stormy, with frequent brief rhythm changes, not however requiring the use of the diving reflex. She died 2 weeks later after a cardiac arrest and necropsy examination showed extensive endocardial fibroelastosis.

The reflex has been used in adults with success (Wildenthal et al., 1975) although periods of immersion of up to 35 seconds have been required. T. Pickering and Bolton-Maggs (1975) reported the risk of ventricular tachycardia being precipitated by this manoeuvre, hence continuous monitoring of the patient during the procedure is advised. The reflex has been used in 2 neonates (Whitman et al., 1977) in whom the response was immediate; as was the case here reported. This reduces the danger of inhalation in an infant. This report show that where standard methods of vagal stimulation have failed to stop a supraventricular tachycardia, and where the use

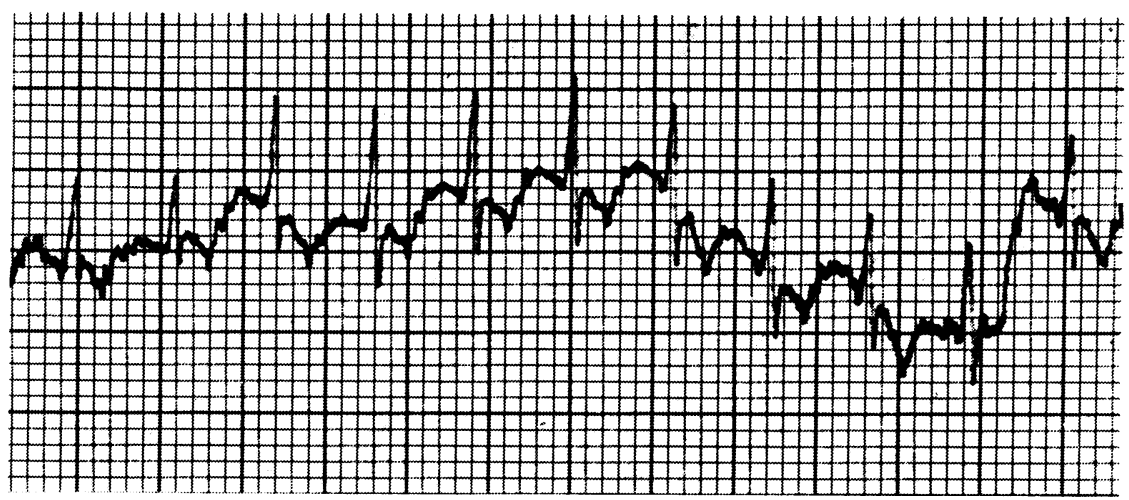

Fig. 1 Standard lead II electrocardiogram before correction of tachycardia.

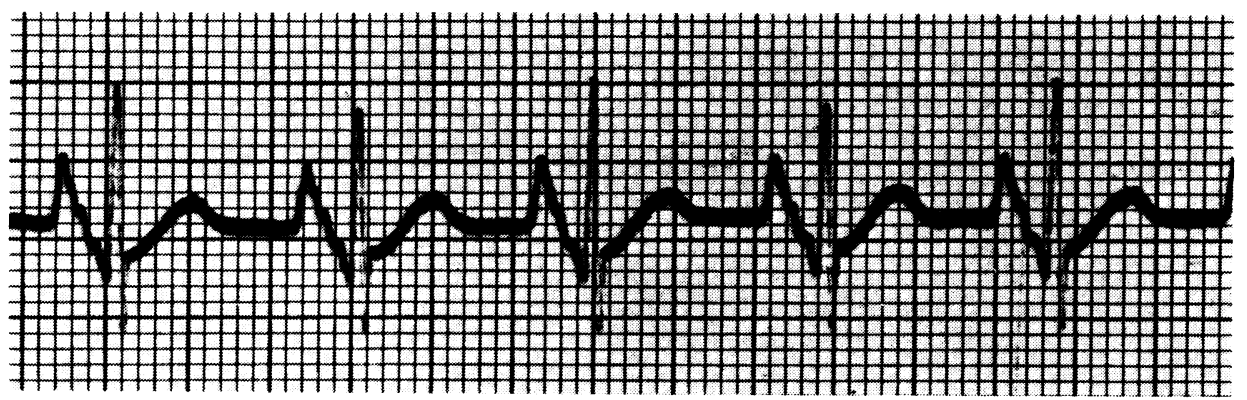

Fig. 2 Standard lead II electrocardiogram immediately following the diving reflex. 
of digoxin makes DC cardioversion more hazardous, the diving reflex may be a useful additional manoeuvre to restore sinus rhythm.

M. F. M. Bamford and Douglas Pickering Department of Paediatrics, The Radcliffe Infirmary, Oxford $O X 26 H E$.

\section{References}

Pickering, T., and Bolton-Maggs, P. (1975). Treatment of paroxysmal supraventricular tachycardia. Lancet, 1, 340.

Whitman, V., and Zakeosian, G. M. (1976). The diving reflex in termination of supraventricular tachycardia in childhood. Journal of Pediatrics, 89, 1032.

Whitman, V., Friedman, L., Berman, W., Jr., and Maisels, M. J. (1977). Supraventricular tachycardia in newborn infants. An approach in therapy. Journal of Pediatrics, 91, 304-305.

Wildenthal, K., Leshin, S. J., Atkins, J. M., and Skelton, C. L. (1975). The diving reflex used to treat paroxysmal atrial tachycardia. Lancet, 1, 12-14.

\section{Hyponatraemia in children with febrile convulsions}

Sir,

Rutter and O'Callaghan (Archives, 1978, 53, 85) have drawn attention to the common finding of mild hyponatraemia in children with febrile convulsions. They define hyponatraemia as plasma $\mathrm{Na}$ levels of $132 \mathrm{mmol} / \mathrm{l}$ and below but do not indicate their reasons for choosing this particular concentration. In our study of 50 healthy babies aged between 18 and 120 days (Dale et al., 1975) we found a mean plasma $\mathrm{Na}$ concentration of $135 \mathrm{mmol} / \mathrm{l}$ (SD 2.3)-similar to the mean value of $135 \mathrm{mmol} / 1$ reported by Rutter and Smales (1977) for 163 children with febrile convulsions. It would seem to be wise, in infancy at least, to take $130 \mathrm{mmol} / \mathrm{l}$ (mean -2SD) as the level below which hyponatraemia is said to exist.

Gordon Dale and J. R. SiberT Department of Clinical Biochemistry, Royal Victoria Infirmary, Newcastle upon Tyne; and Department of Paediatrics, University Hospital of Wales, Cardiff.

\section{References}

Dale, G., Goldfinch, M. E., Sibert, J. R., and Webb, J. K. G. (1975). Plasma osmolality, sodium, and urea in healthy breast-fed and bottle-fed infants in Newcastle upon Tyne. Archives of Disease in Childhood, 50, 731-734.

Rutter, N., and Smales, O. R. C. (1977). Role of routine investigations in children presenting with their first febrile convulsion. Archives of Disease in Childhood, 52, 188-191.

\section{Drs Rutter and O'Callaghan comment:}

We have no information about plasma $\mathrm{Na}$ levels in healthy Nottingham children in the febrile convulsion age group. We therefore defined hyponatraemia as a plasma $\mathrm{Na}$ below the normal ranges quoted by three large children's hospitals: The Hospital for Sick Children, Great Ormond Street (normal range 135-143 mmol/l), Birmingham Children's Hospital (136-143 mmol/l), and Melbourne Children's Hospital (133-143 mmol/l). Our measurements were made on venous blood by flame photometry. Plasma osmolalities, measured by a different method, were also low, suggesting that our low $\mathrm{Na}$ levels were not simply a reflection of our laboratory method.

It may well be that commonly quoted normal ranges for plasma $\mathrm{Na}$ in children are too high, as Dale and Sibert suggest, but we used them in the absence of any other data. We did not use their own values for healthy babies under the age of 4 months because the children in our study were all older, with a mean age of just under 2 years.

\section{N. RutTer and M. J. O'Callaghan Department of Child Health, Nottingham University, and} Nottingham Children's Hospital.

\section{Steroid-induced CPK estimation-a new diagnostic test for human muscular dystrophy and its carrier state}

Sir,

Increase of serum creatine phosphokinase (CPK) has been regarded as the most sensitive index in the detection of early cases of muscular dystrophy, especially in Duchenne type (DMD). Increased CPK is also found in other neuromuscular diseases such as spinal muscular atrophy, motor neurone disease, chronic polymyositis, as well as in $70-75 \%$ of 'definite' carriers of DMD. Takahasi et al. (1975) observed a marked rise of CPK after IV prednisolone in 14 DMD cases and suggested this to be specific for muscular dystrophy (MD), but put forward no explanation for such phenomena.

We have observed the change of CPK in response to IV hydrocortisone in different neuromuscular diseases in Indian patients, so as to confirm or deny Takahasi's findings. Similar testing was also carried out on Duchenne carriers and relatives of other MD cases. 44 cases of MD ( 23 cases of DMD and 21 cases of other MD), 11 cases of other muscle diseases, 6 known Duchenne carriers and 32 suspected carriers were studied. 40 normal controls and 36 healthy relatives of MD cases were also studied.

CPK activity increased significantly 4 hours after $1 \mathrm{mg} / \mathrm{kg}$ IV hydrocortisone in MD cases but not in patients with other neuromuscular disorders. Poststeroid rise in CPK (as \% basal level) was highest in DMD cases (119 \pm 1.78 SD). $50 \%$ of known (definite and probable) Duchenne carriers and $18.7 \%$ of unknown (possible) carriers also showed positive results with the test. No such increase was observed in relatives of MD cases. An inverse correlation was observed between the grade of disability and the \% poststeroid increase of CPK in DMD cases. Such inverse correlation was also found between the duration of disease and poststeroid increase of CPK in DMD cases. The increased CPK may be 